\title{
Approximation Equation for Broad Bandwidth Antenna Design Using CMMS
}

\author{
Kyeong-Sik Min $\cdot$ Ji-Chul Lee
}

\begin{abstract}
This paper proposes an approximate equation for broad bandwidth conditions in an antenna feeding probe design with a cylindrical magneto material structure (CMMS). The bandwidth calculation has been conducted according to the relation between the distance $\left(r_{m}\right)$ between the magneto material and feeding probe, and the magneto material thickness $\left(t_{m}\right)$ for a given $\mu_{r}$. The bandwidth of a proposed antenna with CMM feeding structure is improved about $182 \%$, when $\mu_{r}=20+j 0.001$, in comparison with the bandwidth of an antenna without CMMS. The maximum error extent between the bandwidth calculated by the approximation equation and by the numerical calculation of the proposed antenna is about $\pm 3.2 \%$ for $\mu_{r}=10+j 0.001$. The approximation equation proposed in this study can solve the conventional problem of the complex process and the long time required for reiterative calculation, and allow simple and precise design with prediction. The accuracy of an approximated equation is compared with the results calculated by a commercial tool and verified by reasonable agreement between them.

Key words : CMM, Dipole Antenna Feeding Probe, Broad Bandwidth Condition, Approximation Equation, Calculation Time Improvement.
\end{abstract}

\section{Introduction}

With the recent development of a variety of wireless communication services, the need to include various wireless services in single wireless handheld devices is increasingly stressed [1]. Many antennas with different frequencies and bandwidths are included in a handy terminal to simultaneously provide a variety of wireless communication services. However, this method causes the antenna to take up too much space in the device, and there is the added disadvantage that input impedance matching between the antennas and the RF circuits needs. In order to overcome this disadvantage, a single antenna that can simultaneously catch the frequencies of different bandwidths including multi-band antenna, reconfigurable antenna and broad bandwidth antenna, are currently used. Generally, a multi-band antenna uses a narrow bandwidth element and design is difficult because of sensitive impedance bandwidth and a complex structure [1]. However, when a broad bandwidth antenna is used, there are advantages such as good impedance matching, stable radiation pattern and high efficiency [2]. Antenna types with such broad bandwidth characteristics include the planer monopole antenna, slot antenna, planer oval antenna, PIFA and MDM (Magneto-Dielectric Material). There are many design methods, also. These methods include controlling antenna impedance to match the opera- ting band by changing the antenna structure [3 5], controlling bandwidth with multi-resonance by changing the addition shorting stub or via hole [6, 7], and using dielectric material and magneto material to control the guide wavelength within the dielectric material to ultimately control operation frequency and bandwidth.

In order to reduce the calculation time and to verify accuracy for the model antenna of the reference [8], the approximation equation for the CMM (Cylindrical Magneto Material) feeding structure of the reference [8] has been conducted. The method to control guide wavelength was also considered and applied in this research. The proposed approximation equation is one that can predict conditions of the broad bandwidth according to the relation between the distance $\left(r_{m}\right)$ between the magneto material and feeding probe, and the magneto material thickness $\left(t_{m}\right)$ for each relative permeability $\left(\mu_{r}\right)$. Antenna miniaturization and bandwidth improvement characteristics were designed by adjustment of the parameters of $t_{m}, r_{m}$ and $\mu_{r}$ in the reference [8]. However, because the parameter sensitivity of the CMMS increased to more than $1 / 1,000 \mathrm{~mm}$ by increase of $\mu_{r}$, the materials with high relative permeability were not considered in design.

This sensitivity can require a lot of caution during production and increase the cost of commercial antennas with the CMM structure. Moreover, repeated calculations are required to obtain the optimum parameters for broad

Manuscript received October 19, 2010 ; revised December 7, 2010. (ID No. 20101019-031J)

Department of Radio Communication Engineering, Korea Maritime University, Busan, Korea.

Corresponding Author : Kyeong-Sik Min (e-mail : ksmin@hhu.ac.kr ) 
bandwidth design. Therefore, this study derives the extracted approximation equation for higher reliability as well as fast calculation time in design, and uses the complex relative permeability of magneto material of $\mu_{r}=10+$ $j 0.001$ (low tangential loss magneto material of the magnesium or nickel type), which has less sensitivity in design and is easier and cheaper for production.

\section{Extraction of Approximation Equation for Broad Bandwidth Conditions}

Fig. 1 shows the structure of the vertical feeding dipole antenna used to extract the approximation equation considered in reference [8]. The input impedance $\left(Z_{i n}\right)$ of the antenna was designed as $50 \Omega$, and the structure has the vertical feeding probe in the center of the $\mathrm{Y}$-axis direction as shown in Fig. 1.

As shown in the magnified feeding part of Fig. 1, the CMM completely surrounds the feeding probe.

Fig. 2 shows the examples of the calculated return loss results of using the magneto material with $\mu_{r}=10+$ $j 0.001$ as the CMM structure and applying it to Fig. 1, and calculating the return loss characteristics according to changes of $t_{m}$ and $r_{m}$. The return loss value written by 'Design. ant.' in Fig. 2 means that of the model antenna without CMM of Fig. 1.

The bandwidth is adjusted by the parameters of $t_{m}$ and $r_{m}$, even though $\mu_{r}$ is constant as mentioned in reference [8]. It means that the bandwidth depends on the relation between the $t_{m}$ and $r_{m}$. These parameters largely affect the resistance component and contribute to improvement of return loss. In addition, from the parameter sensitivity perspective, the sensitivity reliability is around $1 / 10 \mathrm{~mm}$ compared to the case of $\mu_{r}=1000+j 0.001$. In order to

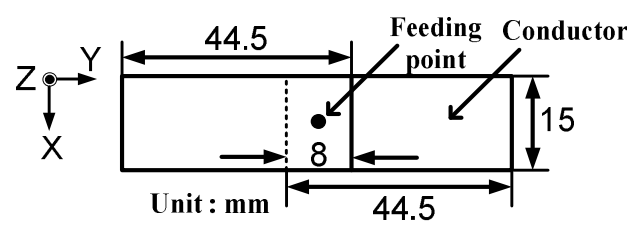

(a) Top view

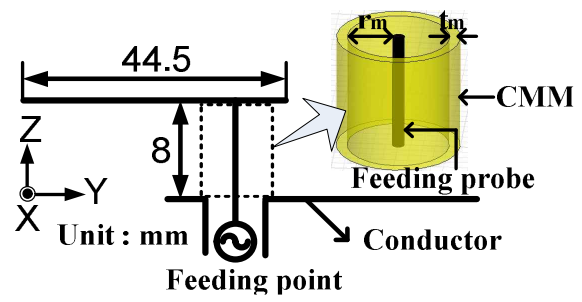

(b) Side view

Fig. 1. Structure of the design antenna without CMM.

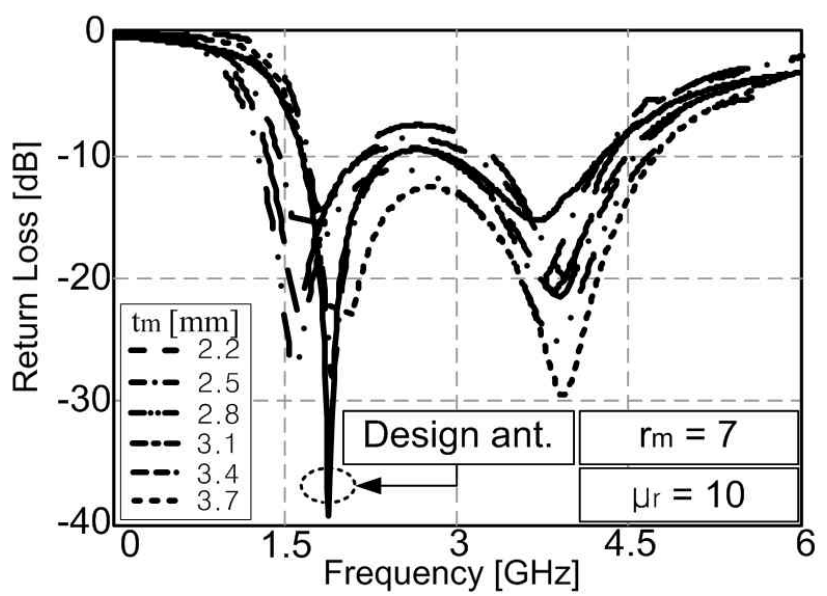

(a) $\mu_{r}=10+j 0.001, r_{m}=7 \mathrm{~mm}$

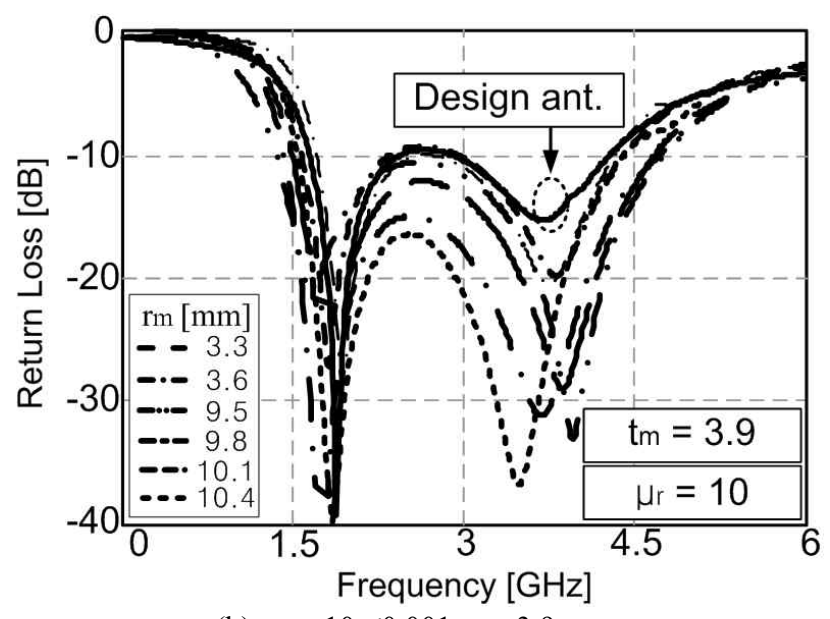

(b) $\mu_{r}=10+j 0.001, t_{m}=3.9 \mathrm{~mm}$

Fig. 2. The calculated return loss according to parameters of $t_{m}$ and $r_{m}$ variation.

find out the relationship between changes in values of $\mu_{r}$, $t_{m}, r_{m}$, and bandwidth, Fig. 3 shows the examples of the calculated return loss characteristics according to a changing value of $\mu_{r}, t_{m}$ and $r_{m}$.

As shown in Fig. 3, when the value of $\mu_{r}$ is $4+$ $j 0.001,20+j 0.001,50+j 0.001$ and $100+j 0.001$ each, the parameter sensitivity of $r_{m}$ increases from $1 / 10 \mathrm{~mm}$ order to $1 / 100 \mathrm{~mm}$ order as the value of $\mu_{r}$ increases. Therefore, the conclusion can be drawn that when a low value of $\mu_{r}$ is used, the sensitivity decreases further than a magneto material with a high $\mu_{r}$ value and makes production easier. In addition, even if the $\mu_{r}$ value changes from 4 to 100 , if $t_{m}$ and $r_{m}$ are adjusted appropriately, there is no large frequency shift, and the broad bandwidth can be designed within the design frequency range. Fig. 4 is a graph that shows the data of Fig. 2 including results in reference [8] and the above data of Fig. 3 in order to examine inter-relation between $t_{m}$ and $r_{m}$ as functions of $\mu_{r}$ for broad bandwidth design. 


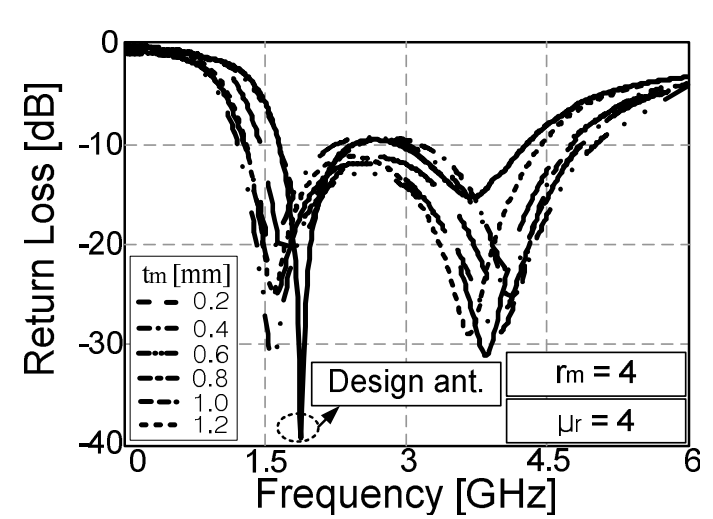

(a) $\mu_{r}=4+j 0.001$ and $r_{m}=4$

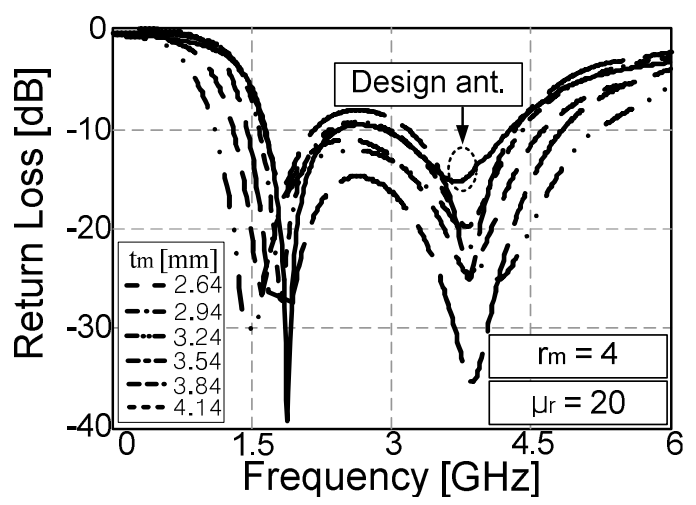

(b) $\mu_{r}=20+j 0.001$ and $r_{m}=4$

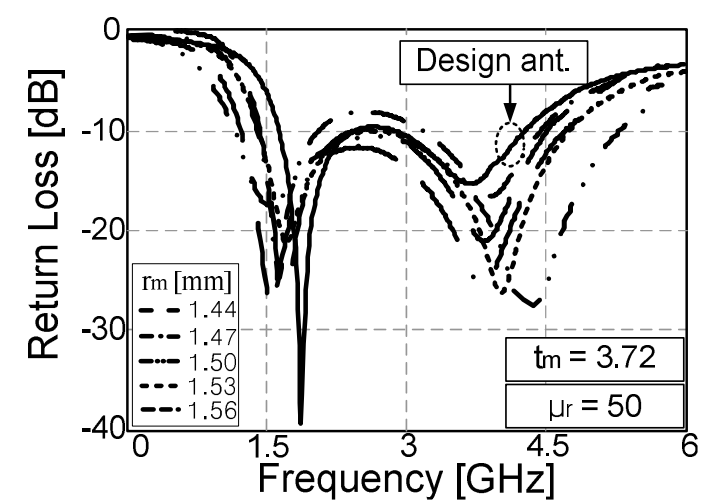

(c) $\mu_{r}=50+j 0.001, t_{m}=3.72$

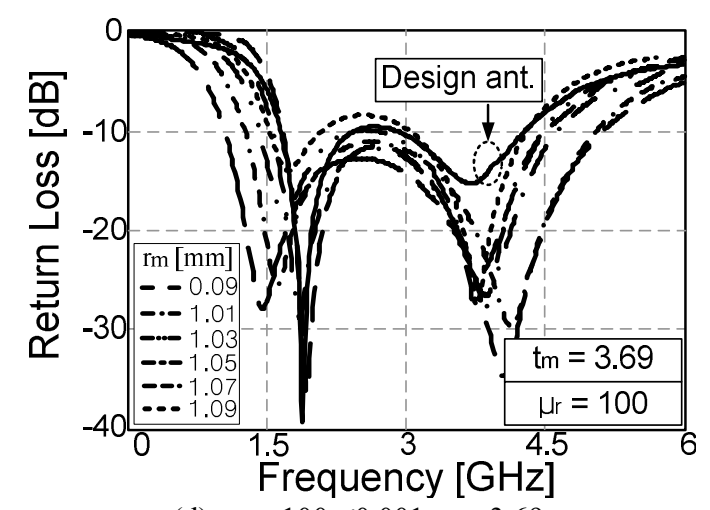

(d) $\mu_{r}=100+j 0.001, t_{m}=3.69$

Fig. 3. The calculated return loss according to variation of $\mu, t_{m}$ and $r_{m}$.

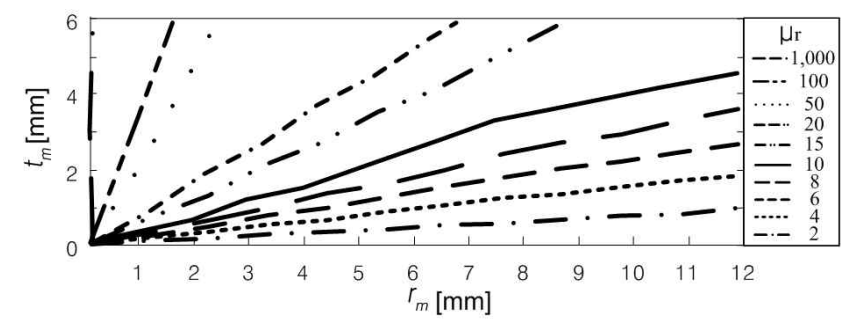

Fig. 4. Relationship of $t_{m}$ and $r_{m}$ with each maximum broad bandwidth characteristics by the calculated return loss.

The method of summarizing data shown in Fig. 4 is as follows. If we take Fig. 2(b), when $\mu_{r}=10+j 0.001, r_{m}=$ $9.5 \mathrm{~mm}, t_{m}=3.9 \mathrm{~mm}$ and the maximum bandwidth has about $4 \mathrm{GHz}$ band, the bandwidth of design antenna without CMMS has remained at about $2.1 \mathrm{GHz}$ band. So these three parameters $\left(\mu_{r}=10+j 0.001, r_{m}=9.5 \mathrm{~mm}, t_{m}=\right.$ $3.9 \mathrm{~mm}$ ) were selected as a set of optimum parameters with the maximum bandwidth as shown in Fig. 4. In order to calculate a set of optimum parameters, much time and repeated calculations were necessary. In the same manner, when $\mu_{r}=50+j 0.001, r_{m}=1.50 \mathrm{~mm}, t_{m}=3.72 \mathrm{~mm}$ and when $\mu_{r}=100+j 0.001, r_{m}=3.69 \mathrm{~mm}, t_{m}=1.03 \mathrm{~mm}$ (Fig. 3(c) and (d)), the maximum bandwidth has about 4.4 GHz and 4.6 GHz band, respectively. These parameters were indicated in Fig. 4.

The magneto's $\mu_{r}$ value was still set at a steady value of 0.001. As shown in Fig. 4, the relationship between $t_{m}$ and $r_{m}$ appears as a linear graph according to changes of $\mu_{r}$. It is dependent on the value of $\mu_{r}$ and has a consistent linear slope $(\theta)$. Therefore, Fig. 4 shows the linear relationship between $t_{m}$ and $r_{m}$ with respect to $\mu_{r}$. Equations (1) and (2) show the approximation equations that can extract a consistent linear slope angle of $\theta$ according to the relationship between $t_{m}$ and $r_{m}$ with respect to $\mu r$.

$$
\begin{aligned}
& t_{m}[\mathrm{~mm}]=0.04 \times \mu_{\mathrm{r}} \times \mathrm{r}_{\mathrm{m}} \\
& \theta=\tan ^{-1}\left(0.04 \times \mu_{r}\right)
\end{aligned}
$$

Where $\theta$ is the linear slope angle made between the $t_{m}$-axis direction and the $r_{m}$-axis direction in Fig. $4,0.04$ is the extracted approximate constant number. The linear slope value $(\theta)$ calculated by equation (2) is directly used as an angle value.

\section{Error Extent Calculation between Numerical Simulation and Approximation}

Fig. 5 shows the error range between the parameters obtained precisely through numerical calculation in Figs. 2 and 3, and from the parameters calculated approxi- 
mately by equations (1) and (2). The numerically simulated calculation results of $\mu_{r}=10+j 0.001$ and $\mu_{r}=20+$ $j 0.001$ from Fig. 4 to extract the error extent in the sample data were considered. The thick solid lines, the dotted lines and the one point chain lines in Fig. 5 represent the numerical calculation results, the average values and the error extent, respectively. The calculation procedure of reaching the error extent to increase reliability of the approximation equation is as follows:

(1) The linear slope angle value $(\theta)$ of the dotted line indicated in Fig. 5 is calculated by equation (2). A straight line draws and becomes the average value. This dotted line of the average value is compared with the numerical calculation value indicated by the thick solid line according to same value of $\mu_{r}$.

(2) Connect the point of the largest numerical calculation value and the point where it meets the straight dotted line that expresses the average value. Apply the same method to the point of smallest numerical calculation value. This line becomes the straight dotted line that expresses extent of error. Lines (a) and (b) are corresponding to these lines in Fig. 5.

(3) In order to increase the reliability of the extracted approximation equation; once the extent of error of each $\mu_{r}$ value is decided, the numerical re-calculation is conducted by using the numbers for $t_{m}$ and $r_{m}$ as shown in Fig. 5. The error extent of the bandwidth computed by the numerical calculation and the approximate equation is compared and analyzed.

When $\mu_{r}=10+j 0.001, \theta=21.8^{\circ}$ by equation (2) as shown in Fig. 5. It is the dotted line that is marked as the average of the $\mu_{r}=10+j 0.001$ group. With this as the standard, if the line that connects the thick solid straight line gained from the numerical calculation with the maximum point that meets the linear slope line (for example, in the graph where $\mu_{r}=10+j 0.001$, the point where $r_{m}=7.5 \mathrm{~mm}$ and $t_{m}=3.3 \mathrm{~mm}$ ) is drawn as $\theta=$ $21.8^{\circ}$, it becomes the straight dotted line a in the figure.

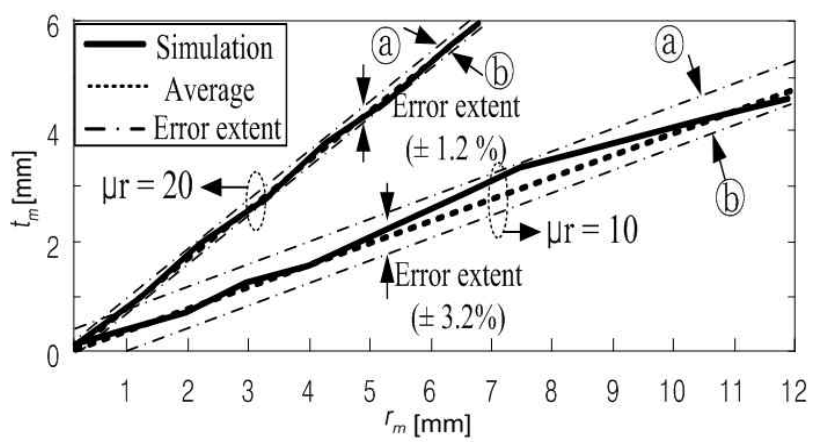

Fig. 5. Example of error extent calculation method using numerical simulation and approximation equation.
In the same way, in the graph where $\mu_{r}=10+j 0.001$, the range up to the lowest point where the line drawn with $\theta=21.8^{\circ}$ (b) meets the point where $r_{m}=12 \mathrm{~mm}$ and $t_{m}=$ $4.4 \mathrm{~mm}$ is regarded as the error extent, and this was shown in Fig. 5. When $\mu_{r}=20+j 0.001, \theta=38.6^{\circ}$ by equation (2), and a graph was drawn in the same way as the method shown above. As a result of extracting the extent of error, when $\mu_{r}=10+j 0.001$ and $\mu_{r}=20+j 0.001$, the extent was $\pm 3.2 \%$ and $\pm 1.2 \%$, respectively, as shown in Fig. 5. In addition, the reason why the bandwidth error extent increases as the value of $\mu_{r}$ increases is because the $\theta$ of the slope equation (2) rapidly increases as shown in Figs. 4 and 5. Also, the values of $r_{m}$ and $t_{m}$ become very sensitive as mentioned in reference [8]. The reason why $\theta$ increases is because the value of $\mu_{r}$ increases by equation (2) and the optimal values of $r_{m}$ and $t_{m}$ come into the very sensitive range of $1 / 1,000 \mathrm{~mm}$ order.

Fig. 6 shows examples of bandwidth error extent by comparing accurate numerical calculation of the $\mu_{r}$ values presented in Fig. 5 and the average value of $\theta$ extracted through the approximation equation. Broad bandwidth appears from the comparison with return loss of Ref. antenna of Fig. 1 without CMMS. Return loss characteristics calculated by equation (1) and (2) are very similar to those derived by precise numerical calculation and have broad bandwidth with very low error. Moreover, when $\mu_{r}$ is given, we can reduce the calculating time to find the optimum parameter of $r_{m}$ and $t_{m}$.

Fig. 7 shows the bandwidth value when it is at the maximum bandwidth through changes in $\mu_{r}$, as shown in Fig. 6 based on results of Fig. 4. When the bandwidth is $100 \%$ in Fig. 7, this indicates that it is a bandwidth range that is calculated when the return loss of a design antenna without CMMS in Fig. 1 is $-10 \mathrm{~dB}$ below. The solid line with black triangle mark shows the results of the bandwidth range through numerical calculation of Fig. 2 and 3, and the dotted line with black circle mark shows the results calculated through the approximation equation (3). Generally, the bandwidth decreases and resonant frequency shifts to low frequency direction when the value of $\mu_{r}$ increases, but as shown in Fig. 6 of reference [8] and Fig. 3 of this study, the bandwidth can increase due to control of the resistance component and the capacitance component of reactance through the detailed adjustment of $r_{m}$ and $t_{m}$ values [9 11]. Therefore, it can be confirmed that the varied optimum parameters of $r_{m}$ and $t_{m}$ values according to change of the $\mu_{r}$ value are more important than the change of only the $\mu_{r}$ value for realization of broad bandwidth.

Equation (3) shows the maximum bandwidth approximation equation for the change in $\mu_{r}$ value, with the 


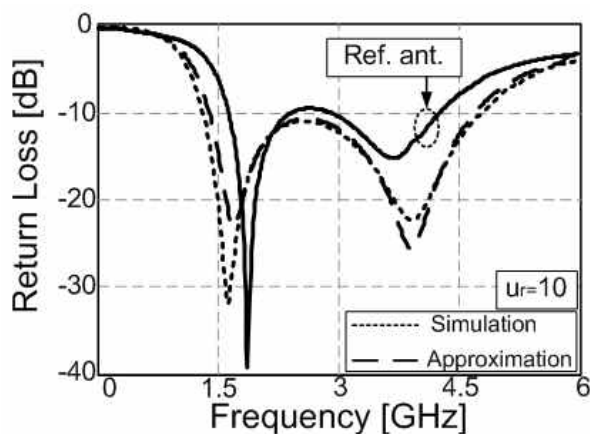

(a) $\mu_{r}=10+j 0.001$

(Sim.: $r_{m}=5, t_{m}=2.1$, Approx.: $\left.r_{m}=5, t_{m}=2\right)$

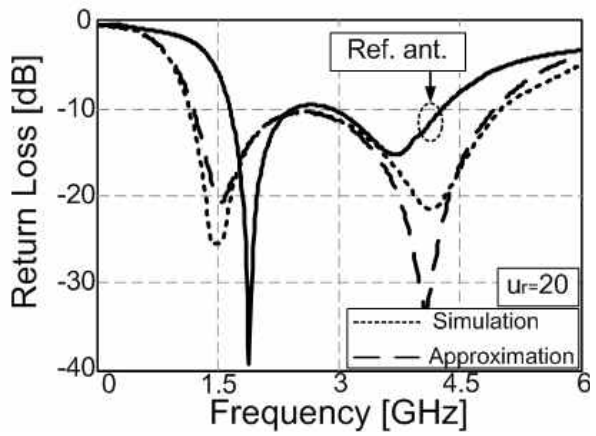

(b) $\mu_{r}=20+j 0.001$

(Sim.: $r_{m}=3, t_{m}=2.7$, Approx.: $r_{m}=3, t_{m}=2.4$ )

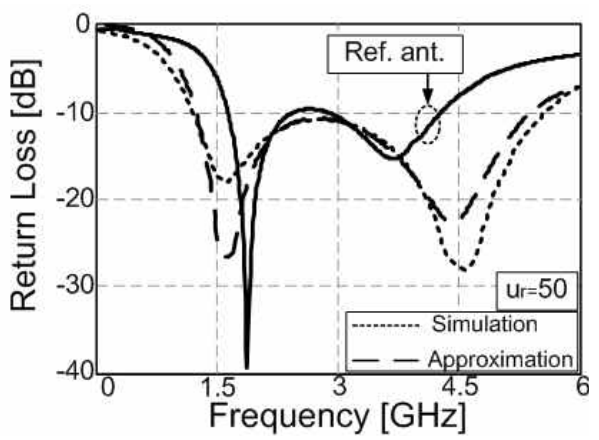

(c) $\mu_{r}=50+j 0.001$

(Sim.: $r_{m}=1, t_{m}=1.91$, Approx.: $r_{m}=1, t_{m}=2$ )

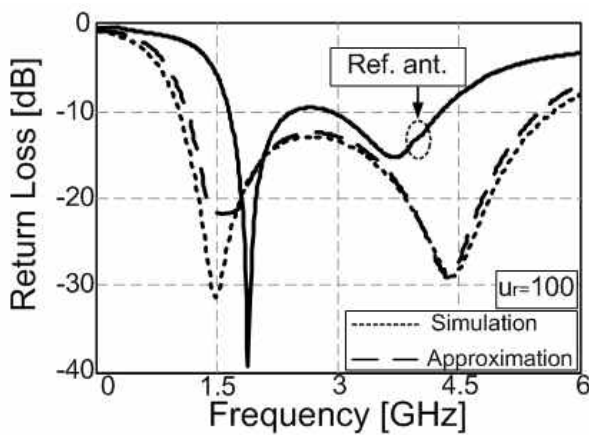

(d) $\mu_{r}=100+j 0.001$

(Sim.: $r_{m}=1, t_{m}=3.84$, Approx.: $r_{m}=1, t_{m}=4$ )

Fig. 6. Comparison of bandwidth error extent between numerically simulated calculation and approximation equation with respect to various $\mu_{r}$ values.

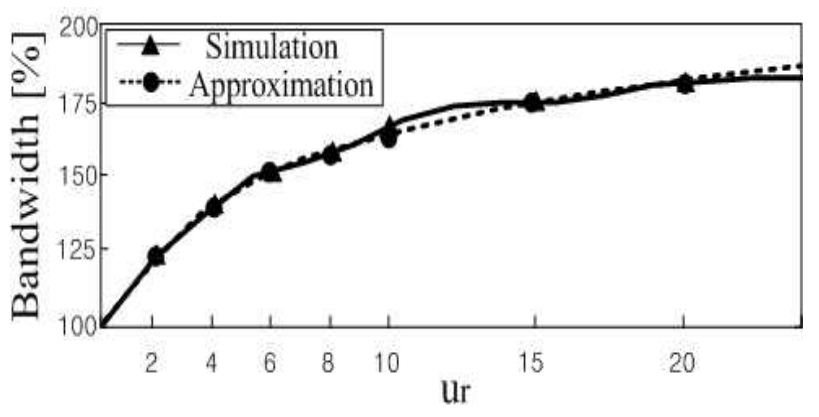

Fig. 7. Maximum bandwidth according to variation of $\mu_{r}$.

bandwidth gained from the numerical calculation in Fig. 7 based on the results of Fig. 4. The optimum values of $t_{m}$ and $r_{m}$ for the given value of $\mu_{r}$ for broad bandwidth design are easily calculated by equation (1) and (2).

Bandwidth $[\%]=28.55 \times \ln \left(\mu_{r}\right)+96.4$

The 28.55 and 96.4 in equation (3) are constants extracted to get a graph closest to the solid line with the black triangle mark obtained from the numerical calculation in Fig. 7.

If the bandwidth range of a design antenna without CMMS in Fig. 1 is set as the standard at $100 \%$, the bandwidth of a design antenna with CMMS is improved about $182 \%$ in comparison with the bandwidth of an antenna without CMMS when $\mu_{r}=20+j 0.001$ as shown in Fig. 7. The maximum error extent between the bandwidth for the approximation of equation (3) and for the numerical calculation of design antenna with CMM feeding structure is around $\pm 3.2 \%$.

Fig. 8 shows the calculated gain directivity pattern with respect to $\mu_{r}=1, \mu_{r}=10$, and $\mu_{r}=20$. Fig. 8(a) of $\mu_{r}=1$ shows the radiation pattern of an antenna without CMMS. The pattern of Fig. 8(b) and (c) is similar to the pattern of Fig. 1(a). However, the calculated gain depends on the value of $\mu_{r}$. When the $\mu_{r}$ increases, the gain decreases because of tangential loss of $\mu_{r}$.

To summarize the results above, the method to extract the appropriate bandwidth for the antenna using CMMS is as follows:

(1) One must determine an appropriate $\mu_{r}$ value with low tangential loss. If the aim is to find the desired bandwidth easily, one can calculate appropriately by using equation (3) for a given $\mu_{r}$ value. When one must determine the $\mu_{r}$ value, equation (3) for the appropriate bandwidth will also be useful as an application method.

(2) Using the calculated $\mu_{r}$ value, the linear slope angles of $t_{m}$ and $r_{m}$ can be calculated using approximation equation (2) and this can be used to find the relationship between $t_{m}$ and $r_{m}$ using equation (1). 


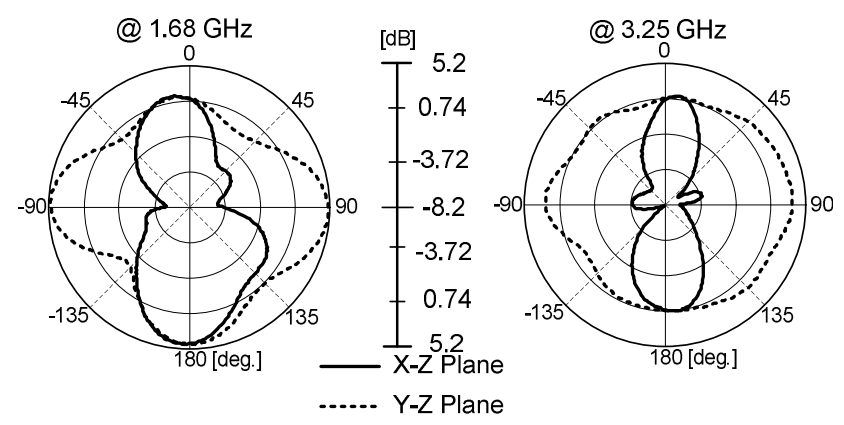

(a) $\mu_{r}=1$

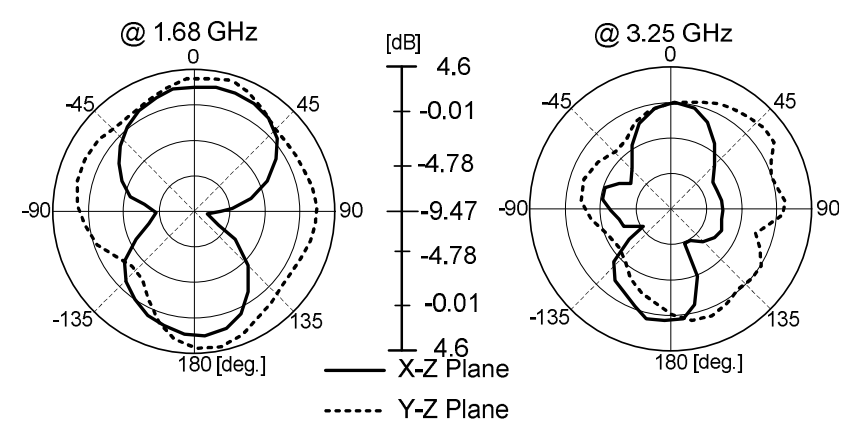

(b) $\mu_{r}=10$

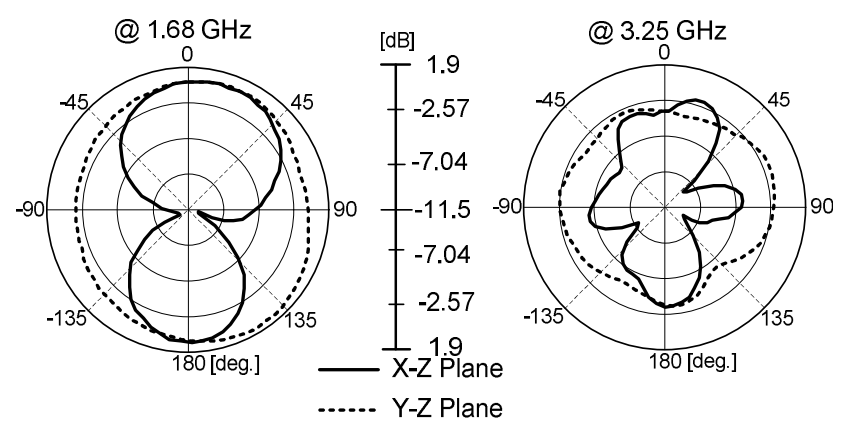

(c) $\mu_{r}=20$

Fig. 8. The calculated gain directivity pattern of the Fig. 1.

(3) One can design a broad bandwidth antenna that has a CMM feeding structure through calculation using the electromagnetic numerical analysis method or the commercial calculation tool for applying the parameters of $\mu_{r}, t_{m}$ and $r_{m}$ derived by the proposed approximation equations.

Conventional calculation to find the optimum parameters of $\mu_{r}, t_{m}$ and $r_{m}$ by using the electromagnetic analysis program including the commercial tools typically requires a complex process and reiterative calculation requiring significant time investment. However, application of the approximation equation proposed in this study can solve the problem of complexity and iterated calculation time, and allow simple and precise design with predictable results.

\section{Conclusion}

This paper presents an approximation equation that allows design of a broad bandwidth antenna by applying a CMM structure at the feeding probe of a dipole type antenna. It confirms that the bandwidth is controlled by adjustment between the distance $\left(r_{m}\right)$ between the cylindrical magneto material and feeding probe, and the magneto material thickness $\left(t_{m}\right)$, even though the resonant frequency is generally shifted dependant on the value of $\mu_{r}$. An approximation equation is derived that can calculate the optimized approximate values with a simple calculation in place of the electromagnetic numerical analysis method or the commercial calculation tools which require a complex process and reiterative calculation that also take a lot of time due to the analysis of $\mu_{r}, t_{m}$ and $r_{m}$ parameters to expand the bandwidth to the maximum. The bandwidth of a proposed antenna with CMM feeding structure is improved about $182 \%$ and the maximum error extent between the bandwidth calculated by the approximation of equation and by the numerical calculation is about $\pm 3.2 \%$ for $\mu_{r}=10+j 0.001$. The approximation equation proposed in this paper can solve the conventional problem of the complex process in calculation and the long time required by reiterative calculation. The reliability and accuracy of an approximated equation were compared with the results calculated by the commercial HFSS tool and verified by reasonable agreement between them.

This research was supported by the Post Brain Korea 21 Project and HCT of Industry Core Technology Development Project by the Ministry of Knowledge Economy.

\section{References}

[1] S. D. Moon, K. Choi, and H. Y. Hwang, "A wideband bow-tie monopole antenna with improved band rejection characteristics," The Journal of Korean Institute of Electromagnetic Engineering and Science, KIEES, vol. 17, no. 12, pp. 1199-1205, Dec. 2006.

[2] W. S. Lee, D. Z. Kim, K. J. Kim, and J. W. Yu, "Multiple frequency notched planar monopole antenna for multiband wireless system," in Proc. 35th Eur. Microw. Conf., Paris, Franch, pp. 1935-1937, Oct. 2005.

[3] H. W Hsieh, Y. C. Lee, K. K. Tiong, and J. S. Sun, "Design of a multiband antenna for mobile handset operations," IEEE Antenna and Wireless Propagation Letter, vol. 8, pp. 200-203, Aug. 2009.

[4] Qinjiang Rao, Wen Geyi, "Compact multiband antenna for handheld device," IEEE Transactions on Antenna and Propagation, vol. 57, no. 10, pp. 3337- 
3339, Oct. 2009.

[5] R. A Bhatti, S. O. Park, "Hepta-band internal antenna for personal communication handsets," IEEE Transactions on Antenna and Propagation, vol. 55, no. 12, pp. 3398-3403, Dec. 2007.

[6] Y. T. J. Charles, V. Ungvichian, "The effects of an additional shorting stub on pifa performance," Asia Pacific Symposium on Electromagnetic Compatibility, Singapore, pp. 558-561, Dec. 2008.

[7] K. J. Oh, Y. T Han, and J. H. Choi, "Design of a compact and broadband pifa using an additive short-circuit plate," The Journal of Korea Information and Communication Society, vol. 28, no. 8, pp. 591-597, Oct. 2003.

[8] J. C. Lee, K. S. Min, "Design of broad bandwidth dipole antenna with CMM feeding probe structure," The Journal of Korean Institute of Electromagnetic Engineering and Science, KIEES, vol. 21, no. 9, pp. 1030- 1036, Sep. 2010.

\section{Kyeong-Sik Min}

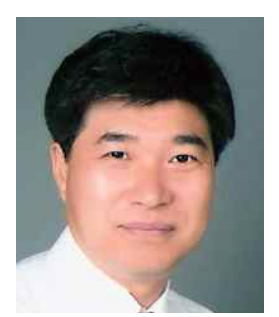

received the B.E. degree in Department of Electronic Communications Engineering from the Korea Maritime University, Korea, in 1989 and 1991, respectively. He received the Ph.D. degree in Department of Electric and Electronics engineering from Tokyo Institute of Technology, Japan, in 1996. Currently, he is professor of the Department of Radio Communication Engineering in Korea Maritime University, Korea. His major research interests include the small planner antenna design for B.S. and mobile communication, the visual design program for microwave circuit \& antenna, small antenna design using Magneto-Dielectric Material, LTE-MIMO antenna and RFID Tag antenna design.
[9] H. Mosallaei, K. Sarabandi, "Antennas with magnetodielectric materials in electro-magnetos: Concept and applications," IEEE Transactions on Antenna and Propagation, vol. 52, no. 6, pp. 1558-1567, Jun. 2004.

[10] M. T. Pekka, S. I. Ikonen, Maslovski, R. S. Constantin, and A. Sergei, "On artificial magnetodielectric loading for improving the impedance bandwidth properties of microstrip antennas," IEEE Transactions on Antenna and Propagation, vol. 54, no. 6, pp. 1654-1662, Jun. 2006.

[11] Douglas J. Kern, Douglas H. Werner, Agostino Monorchio, Luigi Lanuzza, and Michael J. Wilhelm, "The design synthesis of multiband artificial magnetic conductors using high impedance frequency selective surfaces," IEEE Transactions on Antenna and Propagation, vol. 53, no. 1, pp. 8-17, Jan. 2005.

\section{Ji-Chul Lee}

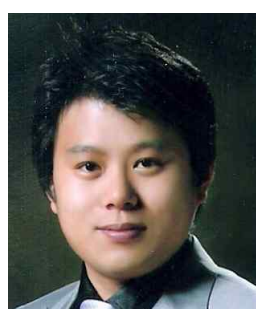

received the B.E. degree and the M.E. degree in Department of Radio Communication Engineering from the Korea Maritime University, Korea, in 2006 and 2008, respectively. Currently, he is antenna engineer of the Ethertronics in Korea. His major research interests include the small planner antenna design for mobile communication system, Broad bandwidth antenna for mobile and wireless communication system, small antenna design using MagnetoDielectric Material and RFID Tag antenna design. 